\title{
歌iresalce
}

\section{Utilização do software ImageJ para avaliar área de lesão dermonecrótica}

\author{
Use of the software ImageJ to evaluate the area of dermonecrotic injury
}

Jaqueline Fernanda Weber ${ }^{1}$, André Luís Fernandes dos Santos ${ }^{2}$

\begin{abstract}
Resumo
Introdução: verificar como se utiliza o software de imagens ImageJ para avaliar área de lesões em processo de dermonecrose em fotografia digital. Métodos: realizou-se revisão da literatura de artigos indexados nas bases de dados BVS/LILACS e PubMed com os descritores DeCS/MeSH, filtros: inglês, português e espanhol; estudos experimentais; 2015, 2017 e 2018. Busca direta no PubMed e seleção de artigos similares. Resultados: foram prospectados 391 artigos na BVS/LILACS, 554 artigos no PubMed e selecionados e analisados 10 artigos. Em dois deles o ImageJ foi utilizado para avaliação do processo cicatricial, um validou o sistema de medição portátil com uso de software para avaliar dimensões de feridas e os demais as ferramentas do ImageJ para processamento de imagens Conclusão: ImageJ se apresentou como importante ferramenta no tratamento e análise quantitativa de imagens, oferecendo muitos recursos como parte de seu pacote padrão e outros tantos como plugins e extensões do software, que se adaptam para cada tipo de uso.
\end{abstract}

Palavras-Chave: Ferimentos e lesões. Processamento de imagem assistida por computador. Software.

\begin{abstract}
Introduction: to verify how to use the free software ImageJ of image analysis to evaluate the area of injury in dermonecrose process in digital photography. Methods: a review of the literature of articles indexed in the databases VHL/LILACS and PubMed with the descriptors DeCS/MeSH, Filters: English, Portuguese and Spanish; Experimental studies; 2015, 2017 and 2018. Direct search in PubMed and selection of similar articles. Results: 391 articles were prospected in the BVS/LILACS, 554 articles in PubMed and selected and analyzed 10 articles. In two of them the ImageJ was used for evaluation of the healing process, one validated the portable measurement system with use of software to assess wound dimensions and the other ImageJ tools for image processing. Conclusion: ImageJ performed as important tool in the treatment and quantitative analysis of images, offering many features as part of your standard package and others as many plugins and software extensions that adapt to each type of use.
\end{abstract}

Keywords: Wounds and injuries. Image processing, computer-assisted. Software.

\footnotetext{
1 Especialista. Centro de Informação e Assistência Toxicológica de Santa Catarina - CIATox/SC/SUR/SES e HU / Universidade Federal de Santa Catarina. Email: jaqueweber@outlook.com

2 Doutor. Fundação Instituto de Educação de Barueri / Universidade Aberta do Brasil - Universidade Federal de São Paulo UNIFESP. Email: andrvet@usp.br

Correspondência: CIATox - Rua Professora Maria Flora Pausewang, s/nº, Campus Universitário - Bairro Trindade. Florianópolis, SC - Brasil. CEP 88036-800.
} 


\section{Introdução}

O loxoscelismo (conjunto de manifestações clínicas decorrentes da inoculação de veneno de aranhas do gênero Loxosceles) é a única causa conhecida de dermonecrose induzida por aranhas $^{1}$. As principais manifestações cutâneas acontecem logo nas primeiras horas em seguida ao acidente, com surgimento característico de lesão apresentando hiperemia, centro isquêmico e edema endurado.

A dermonecrose surge cerca de uma a duas semanas após o acidente, com surgimento de uma lesão ulcerada de cicatrização lenta, eventualmente evoluindo com necessidade de desbridamento cirúrgico².

A ação dermonecrótica do veneno da Loxosceles decorre principalmente da ação das esfingomielinases, presentes apenas em aranhas do gênero Sicarius e em algumas bactérias ${ }^{3}$. Foi demonstrado que as esfingomielinases do veneno de $L$. intermedia induzem a ativação de metaloproteinase (s) endógena (s) no local da inoculação, que foram relacionadas com morte celular ${ }^{4}$.

Demonstrar uma melhoria na cicatrização de lesões está no desfecho da maioria dos estudos. Um desses marcadores indiretos de cura é a taxa percentual de redução da área de seção transversal $^{5}$, a qual pode identificar uma razão para reavaliar a ferida e alterar o tratamento ${ }^{6 ; 7}$.

Somente a avaliação visual da ferida durante o processo de cicatrização é subjetivo e não fornece a área da lesão. $A$ mensuração de área deve ser registrada e comparada com a próxima mensuração, para haver um acompanhamento adequado. Esse registro deve fazer parte da prática clínica rotineira e indispensável nos estudos em cicatrização de feridas ${ }^{5}$.

A área da lesão geralmente é determinada pela multiplicação das duas maiores dimensões (comprimento e largura), e o método tradicional utiliza o traçado do contorno das bordas da ferida em papel quadriculado, de acetato transparente estéril ${ }^{8}$. Todavia, a localização anatômica de algumas feridas pode dificultar o traçado desse contorno ${ }^{7 ; 9}$.

Atualmente, as câmeras digitais presentes nos telefones celulares são acessíveis a muitos profissionais, incluindo médicos e enfermeiros (e às vezes ao paciente e seus parentes), que frequentemente fotografam as lesões para documentar a cicatrização de feridas.

Além disso, as fotografias digitais têm vantagens em relação ao traçado em acetato; porque elas são limpas, evitam o contato com a lesão e economizam tempo durante a avaliação. Entretanto, avaliar 
uma fotografia é subjetivo e não tem valor algum sem a mensuração da lesão ${ }^{9}$.

No mercado existem vários softwares para análise de imagens, porém são muito caros e sua utilização complexa necessita de treinamento específico. Por isso, é interessante o uso de um software livre, que acompanhe um manual de auxílio ao usuário e o estabelecimento de rotinas de análises, que atendam às necessidades dos diversos grupos de pesquisa.

Esse estudo objetiva verificar na literatura, como se utiliza o software livre de análise de imagens ImageJ, para avaliar área de lesões em processo de dermonecrose, em fotografia digital.

\section{Métodos}

Trata-se de uma revisão narrativa, ou seja, um estudo secundário de revisão da literatura sobre o uso do software ImageJ, para avaliar área de lesão dermonecrótica. Foram utilizados, como fonte de dados, periódicos indexados na BVS/LILACS e PubMed.

Os critérios de seleção incluíram a busca por artigos publicados nos anos 2017 e 2018, em português, espanhol e inglês, estudos experimentais, com descritores: ferimentos e lesões; "wounds and injuries"; "heridas y lesiones"; cicatrização; "wound healing"; "cicatrización de heridas"; fotografia; "photography"; pele; "skin"; "piel"; processamento de imagem assistida por computador; "image processing, computerassisted"; "procesamiento de imagen assistida por computador"; imagej; "software"; "user-computer interface"; imagem com lapso de tempo; "time-lapse imaging"; "imagen de lapso de tiempo"; planimetria digital; "digital planimetry"; sensibilidade e especificidade; "sensitivity and specificity"; "sensibilidade y especificidad"; "clinical trial", excluindo-se todos os que não se referiam ao software ImageJ.

Os critérios de seleção dos estudos também contemplaram artigos sem indexação (busca direta) no PubMed e posterior busca nos artigos similares, esses com período de abrangência nos anos 2015, 2017 e 2018.

Os artigos foram analisados buscando relação entre o uso do software ImageJ, como técnica de mensuração e avaliação de lesões.

\section{Resultados}

Foram analisados 391 artigos na BVS/LILACS, dos quais dois abordaram a relação entre o uso do software ImageJ, como técnica de mensuração e avaliação de lesões ${ }^{8: 9}$ e um valida o sistema de medição portátil com uso de software, para avaliar dimensões de feridas ${ }^{7}$. Os demais artigos utilizam outras técnicas de processamento de imagem assistida por computador ou outro software, como métodos para mensuração e avaliação de 
dados nas ciências biológicas ou da saúde (Quadro 1), disponível nos anexos.

No PubMed foram 554 artigos e nenhum utilizou o ImageJ para avaliar área de lesões. Diante disso, foi realizada busca direta de artigos não indexados com a palavra-chave ImageJ e retornaram quatro artigos, sendo dois desses relacionados com o uso do ImageJ para processamento de imagens ${ }^{10,11}$. Na busca por artigos similares aos dois, obtiveram-se 116 artigos e cinco foram selecionados $^{12,13,14,15,16}$; os demais estudos são anteriores ao ano de 2013 ou possuem outra abordagem fora do escopo da pesquisa (Quadro 1), disponível nos anexos.

\section{Discussão}

O ImageJ tem sido utilizado para medir diferentes imagens científicas e médicas, incluindo desordens cutâneas. $^{8,10,13,15}$ Jeffcoate et al (2017) estudaram a mensuração da área transversal de úlceras em pé diabético usando Image ${ }^{5}$. No entanto, as imagens digitais não eram exatamente das fotografias das feridas, mas do traçado da borda da úlcera em acetato estéril. Essa metodologia utiliza folha com quadrados de grade e a área da lesão é determinada pela contagem dos quadrados. Entretanto, o cálculo da área não é suficientemente preciso, pois além de atribuir um formato retangular, atualmente a folha não possui quadriculados menores que $1,0 \times 1,0 \mathrm{~cm}$. Aragón-Sánchez et al (2017) mediram imagens em fotografias digitais e consideraram algumas vantagens sobre 0 traçado em acetato, já que as mesmas permitem uma captura rápida e limpa, então há economia de tempo durante a avaliação, não há contato com a lesão, além de favorecer o tratamento ao salvar o valor mensurado em banco de dados digital e possibilitar a comparação das áreas ${ }^{8}$.

Outro artigo, de Turin et al (2018), compara a análise digital com os métodos tradicionais e essa fornece medidas mais confiáveis e precisas da espessura dérmica e epidérmica, pois as amostras de pele no formato retângulo compensam os contornos tortuosos da junção epidérmicodérmica ${ }^{9}$.

No entanto, Aragón-Sánchez et al (2017) afirmam que medir área curva com - ImageJ pode perder precisão quando a úlcera se estender ao redor da perna, nessa situação, o traçado em acetado estéril poderia ser uma solução aceitável para o problema ${ }^{8}$. Outra questão seria o ângulo da câmera ao tirar as fotografias. O mesmo autor relata em seu estudo que a câmera sempre foi colocada em paralelo com a régua e as fotos foram tiradas com o iPhone 6S (Apple Inc., Cupertino, CA), uma câmera de 12 megapixels usando o flash, contudo poderia ser qualquer outra câmera digital e isso não traria diferenças significativas ${ }^{8}$.

Aragón-Sánchez et al (2017), em sua pesquisa, demonstraram como utilizaram o ImageJ para avaliar área de lesões. 
Explicam que as fotografias foram tiradas com uma régua ao lado da ferida e em paralelo com a pele saudável (Figura 1, 2 e 3), disponíveis nos anexos. As imagens originais foram copiadas em arquivos dentro de uma pasta (formato JPG) e na sequência visualizadas com o Image $J^{8,17}$. Como realizou:

1. Abriu o ImageJ e clicou em File>Open (arrastar e soltar a imagem no software é uma alternativa).

2. Desenhou um segmento ao longo da régua, usando a ferramenta "straight line" de $1 \mathrm{~cm}$ (Figura 1). O software calcula a distância em pixels.

3. Analyze menusset scale>known distance (usou 10) > unit of length (mm). O software automaticamente recalcula o número de pixels $/ \mathrm{mm}$.

4. O contorno da ferida foi criado usando a ferramenta "Freehand selections" com o mouse do computador (Figura 2).

5. Analyse menusmeasure. A área em $\mathrm{mm}^{2}$ foi então calculada.

6. Captura de tela com a janela de medição foi salva no banco de dados (Figura 3).

Finalmente, o ImageJ, em linguagem Java (desenvolvido por Wayne Rasband do Research Services Branch, National Institute of Mental Health, Bethesda, Maryland) possibilita o desenvolvimento de plugins, em menu conveniente, que se adequam às necessidades dos usuários. Suporta várias extensões (TIF/TIFF, BMP, JPG/JPEG, PGM, DICOM, FITS, NIH), linguagem de Script (Macros) em JAVA com comandos do próprio software.

Disponibiliza grande quantidade de filtros e operações para processamento, com permissões de download e instalação de filtros desenvolvidos por outros usuários participantes de fóruns do software. Possui comando que permite salvar arquivo de imagens em texto (formato TXT) e exportar a janela contendo os resultados em formato XLS. Além de tutoriais e vários fóruns de ajuda online ${ }^{10,17}$.

\section{Conclusão}

O software ImageJ se apresentou como uma importante ferramenta no tratamento de imagens e análise quantitativa de imagens, oferecendo muitos recursos como parte de seu pacote padrão e outros tantos como plugins e extensões do software, que se adaptam para cada tipo de uso. O fato de possuir mailing list e ser um software de licença livre estimula diversos grupos de discussão e desenvolvimento de novas ferramentas, que aprimoram o software. A documentação existente no site do software é de muita ajuda ao usuário, pois explica bem o seu uso. Em conclusão, o processamento de imagem assistida por computador utilizando o ImageJ estima a área de ferimentos e lesões, em fotografia digital, com excelente confiabilidade. Ao paciente e aos profissionais da saúde, é uma maneira gratuita, rápida e precisa de medição, a qual poderá ser utilizada 
rotineiramente na prática clínica, para documentar a cicatrização de feridas.

\section{Referências}

1. SWANSON, David L.; VETTER, Richard S. Loxoscelism. Clinics in Dermatology, Philadelphia, vol.24 (3), pp.213-221, jun. 2006. Disponível em: <https://www.sciencedirect.com/science/ar ticle/pii/S0738081X05001720?via\%3Dihu b>. Acesso em: 03 nov. 2018.

2. BRASIL. Ministério da Saúde. Coordenação de Vigilância das Doenças Transmitidas por Vetores e Antropozoonoses e Fundação Nacional de Saúde. Manual de diagnóstico e tratamento de acidentes por animais peçonhentos. 2. ed. Brasília: Fundação Nacional de Saúde, 2001. 120 p. Disponível em:

<http://bvsms.saude.gov.br/bvs/publicaco es/funasa/manu_peconhentos.pdf> Acesso em: 03 nov. 2018.

\section{YOUNG, Anna R.; PINCUS Steven J.} Comparison of enzymatic activity from three species of necrotizing arachnids in Australia: Loxosceles rufescens, Badumna insignis and Lampona cylindrata. Toxicon, London, vol. 39(2-3), pp.391-400, feb./mar. 2000. Disponível em: <

https://www.sciencedirect.com/science/arti cle/pii/S0041010100001458?via\%3Dihub > Acesso em: 03 nov. 2018.

4.TAMBOURGI, Denise V.; PAIXÃOCAVALCANTE, D.; GOLÇALVES DE ANDRADE, Rute M.; FERNANDESPEDROSA, Matheus de F.; MAGNOLI, Fábio C.; PAUL MORGAN, B.; VAN DEN BERG, Carmen W. Loxosceles sphingomyelinase induces complementdependent dermonecrosis, neutrophil infiltration, and endogenous gelatinase expression. Jornal of Investigative Dermatology, London, vol. 124(4), pp.72531, apr. 2005. Disponível em: < https://www.sciencedirect.com/science/arti cle/pii/S0022202X15322363> Acesso em: 03 nov. 2018.
5. JEFFCOATE, William J.; MUSGROVE, Alison J.; LINCOLN, Nadina B. Using image $\mathrm{J}$ to document healing in ulcers of the foot in diabetes. International Wound Journal, Nottingham, vol. 14(6), pp.11371139, dec. 2017. Disponível em: $<$ https://onlinelibrarywiley.ez69.periodicos.capes.gov.br/doi/ep df/10.1111/iwj.12769 >. Acesso em: 02 nov. 2018.

6. LAVERY, Lawrence A.; BARNES, Sunni A.; KEITH, Michael S.; SEAMAN, John W.; ARMSTRONG, David G. Prediction of healing for postoperative diabetic foot wounds based on early wound area progression. Diabetes care, Virginia, vol. 31(1), pp.26-9, jan. 2008.

Disponível em: < http://care.diabetesjournals.org/content/31 /1/26\#ref-1>. Acesso em: 02 nov. 2018.

7. PAVLOVČIČ, U.; JEZERŠEK, M. Handheld 3-dimensional wound measuring system. Skin Research and Technology, London, vol. 24(2), pp.326333, may. 2018. Disponível em: <http://pesquisa.bvsalud.org/portal/resour $\mathrm{ce} / \mathrm{pt} / \mathrm{mdl}-29377336>$. Acesso em: 07 out. 2018.

8. ARAGÓN-SÁNCHEZ, Javier; QUINTANA-MARRERO, Yurena; ARAGÓN-HERNÁNDEZ, Cristina; HERNÁNDEZ-HERERO, María José. ImageJ: A Free, Easy, and Reliable Method to Measure Leg Ulcers Using Digital Pictures. The International Journal of Lower Extremity Wounds, London, vol. 16(4), pp. 269-273, dec. 2017. Disponível em: <

http://pesquisa.bvsalud.org/portal/resourc e/pt/mdl-29251541>. Acesso em: 07 out. 2018.

9. TURIN, Sergey Y.; LEDWON, Joanna K.; BAE, Hanna; BUGANZA-TEPOLE, Adrian; TOPCZEWSKA, Jolanta; GOSAIN, Arun K.; Digital analysis yields more reliable and accurate measures of dermal and epidermal thickness in histologically processed specimens compared to traditional methods.

Experimental Dermatology, Austrian, vol. 27(6), pp.687-690, jun. 2018. Disponível em: < 
http://pesquisa.bvsalud.org/portal/resourc e/pt/mdl-29573291 >. Acesso em: 07 out. 2018.

10. HINER, Mark C.; RUEDEN, Curtis T.; ELICEIRI, Kevin W. ImageJ-MATLAB: a bidirectional framework for scientific image analysis interoperability. Bioinformatics, Oxford, vol. 33(4), pp.629-630, Feb. 2017. Disponível em:

<https://www.ncbi.nlm.nih.gov/pubmed/27 797782>. Acesso em: 07 out. 2018.

11. RUEDEN, Curtis T.; SCHINDELIN, Johannes; HINER, Mark C.; DEZONIA, Barry E.; WALTER, Alison E.; ARENA, Ellen T.; ELICEIRI, Kevin W. ImageJ2: ImageJ for the next generation of scientific image data. Bioinformatics, London, vol. 18(1), nov. 2017. Disponível em: <https://www.ncbi.nlm.nih.gov/pubmed/29 187165>. Acesso em: 07 out. 2018.

12. SCHINDELIN, Johannes; RUEDEN, Curtis T.; HINER, Mark C.; ELICEIRI, Kevin W. The ImageJ ecosystem: An open platform for biomedical image analysis. Molecular Reproduction \& Development, New York, vol. 82, pp. 518529, jul. 2015. Disponível em:

$<$ https://www.ncbi.nlm.nih.gov/pubmed/26 153368 >. Acesso em: 07 out. 2018.

13. NANES, Benjamin A. Slide Set: Reproducible image analysis and batch processing with ImageJ. Biotechniques, London, vol. 59(5), pp. 269-278, may.
2016. Disponível em:

$<$ https://www.ncbi.nlm.nih.gov/pubmed/26

554504>. Acesso em: 07 out. 2018.

14. DOMÍNGUEZ, César; HERAS, Jónathan; PASCUAL, Vico. IJ-OpenCV: Combining ImageJ and OpenCV for processing images in biomedicine. Computers in Biology and Medicine, New York, vol. 84, pp.189-194, may. 2017. Disponível em: $<$ https://www.ncbi.nlm.nih.gov/pubmed/28 390286>. Acesso em: 07 out. 2018.

15. DELLA MEA, Vincenzo; BARONI, Giulia L.; PILUTTI, David; DI LORETO, Carla. SlideJ: An ImageJ plugin for automated processing of whole slide images. PLoS ONE, Austrian, vol. 12(7), p. e0180540, jul. 2017. Disponível em: $<$ https://www.ncbi.nlm.nih.gov/pubmed/28 683129>. Acesso em: 07 out. 2018.

16 HARRINGTON, Kyle I. S.; RUEDEN, Curtis T.; ELICEIRI, Kevin W. FunlmageJ: A Lisp framework for scientific image processing. Bioinformatics, Oxford, vol. 34(5), pp. 899-900, nov. 2017. Disponível em:

$<$ https://www.ncbi.nlm.nih.gov/pubmed/29 106446>. Acesso em: 07 out. 2018.

17. RASBAND, W. Image J documentation. Disponível em: $<$ http://rsb.info.nih.gov/ij/docs/index.html>. Acesso em: 09 nov. 2018. 


\section{Anexos}

Quadro 1 - Artigos localizados na BVS/LILACS (2017 e 2018) e PUBMED (2015, 2017 e 2018), sobre a relação entre o uso do software ImageJ como técnica de mensuração e avaliação de lesões.

\begin{tabular}{|c|c|c|}
\hline $\begin{array}{c}\text { Título do } \\
\text { Artigo/Referência }\end{array}$ & Objetivo & Resultados \\
\hline $\begin{array}{l}\text { ImageJ: a free, easy, } \\
\text { and reliable method to } \\
\text { measure leg ulcers } \\
\text { using digital pictures }^{8}\end{array}$ & $\begin{array}{l}\text { Documentar o curso de } \\
\text { cicatrização das úlceras } \\
\text { crônicas das pernas por } \\
\text { mensuração da ferida. }\end{array}$ & $\begin{array}{l}\text { A análise de imagens digitais de } \\
\text { úlceras de perna com o software } \\
\text { ImageJ estima a área da ferida com } \\
\text { excelente confiabilidade. Esse } \\
\text { método gratuito, rápido e preciso } \\
\text { poderia ser usado rotineiramente na } \\
\text { prática clínica para documentar a } \\
\text { cicatrização de feridas. }\end{array}$ \\
\hline $\begin{array}{l}\text { Digital analysis yields } \\
\text { more reliable and } \\
\text { accurate measures of } \\
\text { dermal and epidermal } \\
\text { thickness in } \\
\text { histologically processed } \\
\text { specimens compared to } \\
\text { traditional methods }\end{array}$ & $\begin{array}{l}\text { Verificar o método mais confiável } \\
\text { e preciso para medir espessura } \\
\text { em camadas dérmicas e } \\
\text { epidérmicas na pele. }\end{array}$ & $\begin{array}{l}\text { O uso da microscopia acoplada a um } \\
\text { software de captura de imagens } \\
\text { capaz de criar uma imagem digital da } \\
\text { lâmina a ser analisada pelo software } \\
\text { ImageJ, apresentou-se como um } \\
\text { método preciso, didático e confiável } \\
\text { para medir a espessura das camadas } \\
\text { da pele. Estas no formato de } \\
\text { retângulo compensaram os contornos } \\
\text { tortuosos da junção epidérmico- } \\
\text { dérmica. }\end{array}$ \\
\hline 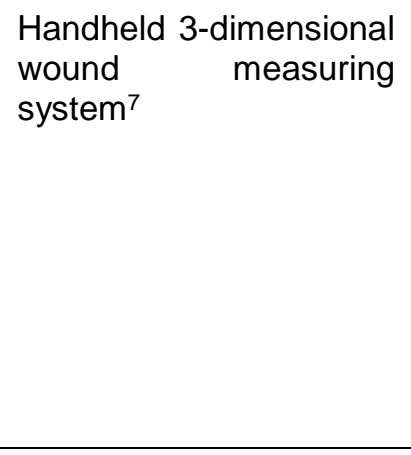 & $\begin{array}{l}\text { Validar um sistema de medição } \\
\text { portátil das dimensões de } \\
\text { feridas, com o uso de um } \\
\text { software adequado. }\end{array}$ & $\begin{array}{l}\text { A medição em três dimensões, com } \\
\text { equipamento portátil de projeção } \\
\text { estruturada de luz permite uma } \\
\text { captura da ferida e análise de cor da } \\
\text { borda pelo software. Tal sistema } \\
\text { apresentou resultados rápidos, } \\
\text { reprodutíveis e representativos. O } \\
\text { método possui limitações devido aos } \\
\text { diferentes volumes das feridas e } \\
\text { poderá subestimar camadas mais } \\
\text { superficiais. }\end{array}$ \\
\hline $\begin{array}{l}\text { ImageJ-MATLAB: a } \\
\text { bidirectional framework } \\
\text { for scientific image } \\
\text { analysis } \\
\text { interoperability }{ }^{10}\end{array}$ & $\begin{array}{l}\text { Adicionar na área de trabalho do } \\
\text { computador um software de } \\
\text { estrutura bidirecional para } \\
\text { interoperabilidade e análise de } \\
\text { imagem. }\end{array}$ & $\begin{array}{l}\text { O software ImageJ-MATLAB é uma } \\
\text { biblioteca Java que possibilita a } \\
\text { interoperabilidade bidirecional entre o } \\
\text { MATLAB e o ImageJ. Ao definir um } \\
\text { padrão de tradução entre estruturas } \\
\text { de dados matriciais e imagens, } \\
\text { permite selecionar ferramentas } \\
\text { personalizadas para a análise de } \\
\text { imagens. }\end{array}$ \\
\hline $\begin{array}{l}\text { ImageJ2: ImageJ for the } \\
\text { next generation of } \\
\text { scientific image data }{ }^{11}\end{array}$ & $\begin{array}{l}\text { O ImageJ2 } \text { como uma } \\
\text { ferramenta para reestruturar o } \\
\text { ImageJ e permitir } \\
\text { funcionalidade, extensibilidade, } \\
\text { reprodutibilidade, usabilidade e } \\
\text { desempenho. }\end{array}$ & $\begin{array}{l}\text { Transforma o software ImageJ num } \\
\text { aplicativo flexível e com novas } \\
\text { funcionalidades para análise e } \\
\text { processamento de imagens, além de } \\
\text { uma estrutura com interoperabilidade } \\
\text { entre os programas externos. }\end{array}$ \\
\hline
\end{tabular}

The ImageJ ecosystem: An open platform for biomedical image analysis $^{12}$
Estudar como um software livre, Com o advento do ImageJ2 as ImageJ, promove um funcionalidades de macros e plugins ecossistema com ferramentas foram facilitadas e podem ser que facilitam a análise de acessadas a partir de outros 


\begin{tabular}{|c|c|c|}
\hline & $\begin{array}{l}\text { imagens pela comunidade } \\
\text { científica. }\end{array}$ & $\begin{array}{l}\text { aplicativos. Essa interoperalidade } \\
\text { fornece interfaces unificadas para } \\
\text { manipulações de imagens e nos } \\
\text { próximos anos irá avançar no } \\
\text { desenvolvimento de um software } \\
\text { científico para apoio à pesquisa. }\end{array}$ \\
\hline $\begin{array}{l}\text { Slide Set: Reproducible } \\
\text { image analysis and } \\
\text { batch processing with } \\
\text { Image }{ }^{13}\end{array}$ & $\begin{array}{l}\text { Verificar um software para } \\
\text { análise de imagens, conectado } \\
\text { com o ImageJ, que permita } \\
\text { reprodutibilidade, } \\
\text { processamento e organização } \\
\text { de um grande conjunto de dados } \\
\text { em tabelas. }\end{array}$ & $\begin{array}{l}\text { O Slide Set organiza imagens e dados } \\
\text { em tabelas, o que melhora eficiência e } \\
\text { reprodutibilidade na análise de } \\
\text { imagens, devido à automatização de } \\
\text { plugins para executar tarefas simples } \\
\text { e repetitivas. }\end{array}$ \\
\hline $\begin{array}{l}\text { IJ-OpenCV: Combining } \\
\text { ImageJ and OpenCV for } \\
\text { processing images in } \\
\text { biomedicine }^{14}\end{array}$ & $\begin{array}{l}\text { Conectar dois softwares com } \\
\text { funcionalidades diferentes, } \\
\text { porém complementares para } \\
\text { análise de imagens: o ImageJ } \\
\text { com uma biblioteca } \\
\text { multiplataforma para o } \\
\text { desenvolvimento de aplicativos } \\
\text { na área de visão computacional, } \\
\text { o OpenCV. }\end{array}$ & $\begin{array}{l}\text { A biblioteca multiplataforma OpenCV } \\
\text { contribuiu na construção de vários } \\
\text { plugins, os quais favoreceram a } \\
\text { interoperabilidade com o software } \\
\text { ImageJ. Além disso, futuras versões } \\
\text { da biblioteca ImageJ-OpenCV } \\
\text { pretendem empregar novos recursos } \\
\text { para maior extensibilidade com outros } \\
\text { sistemas. }\end{array}$ \\
\hline $\begin{array}{l}\text { SlideJ: An ImageJ } \\
\text { plugin for automated } \\
\text { processing of whole } \\
\text { slide images }^{15}\end{array}$ & $\begin{array}{l}\text { Utilizar o plugin SlideJ com o } \\
\text { objetivo de analisar imagens de } \\
\text { campo microscópico, capturadas } \\
\text { em slide digital. }\end{array}$ & $\begin{array}{l}\text { O SlideJ fornece um macro para } \\
\text { processar automaticamente slides } \\
\text { digitais no ImageJ, o que permite uma } \\
\text { análise de slides em bloco. No } \\
\text { momento nenhum outro plugin } \\
\text { fornece essa função. }\end{array}$ \\
\hline $\begin{array}{l}\text { FunlmageJ: a Lisp } \\
\text { framework for scientific } \\
\text { image processing }\end{array}$ & $\begin{array}{l}\text { O FunlmageJ como uma } \\
\text { biblioteca multiplataforma na } \\
\text { área de visão computacional, } \\
\text { para facilitar a interoperabilidade } \\
\text { entre linguagem artificial e } \\
\text { análise de imagens pelo ImageJ. }\end{array}$ & $\begin{array}{l}\text { O FunlmageJ oferece uma } \\
\text { programação para análise de imagens } \\
\text { biológicas e permite processar } \\
\text { imagens utilizando funções } \\
\text { matemáticas, com conservação dos } \\
\text { dados. }\end{array}$ \\
\hline
\end{tabular}

Fonte: os autores

\section{Lista de figuras}

Figura 1: Desenhou um segmento ao longo da régua usando a ferramenta "straight line".

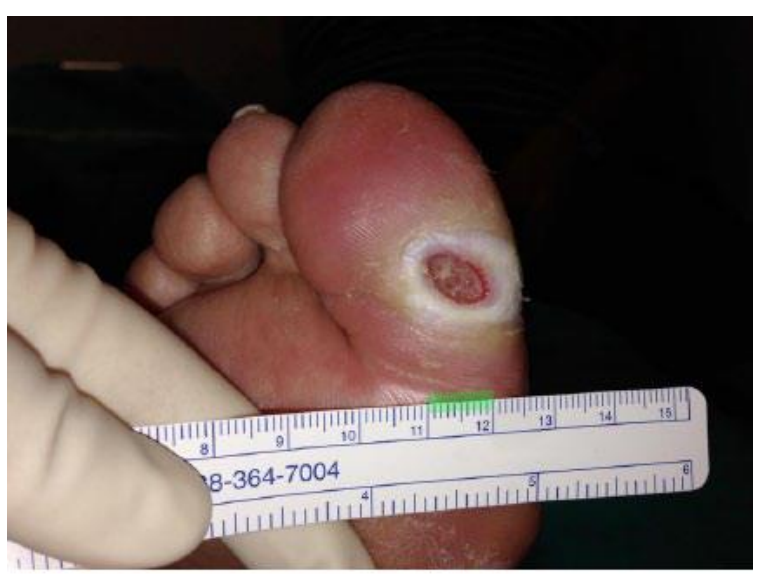

Fonte: Adaptado de Aragón-Sánchez et al., 2017). 
Figura 2: Criou o contorno da ferida usando a ferramenta "Freehand selections" e traçou o formato da ferida.

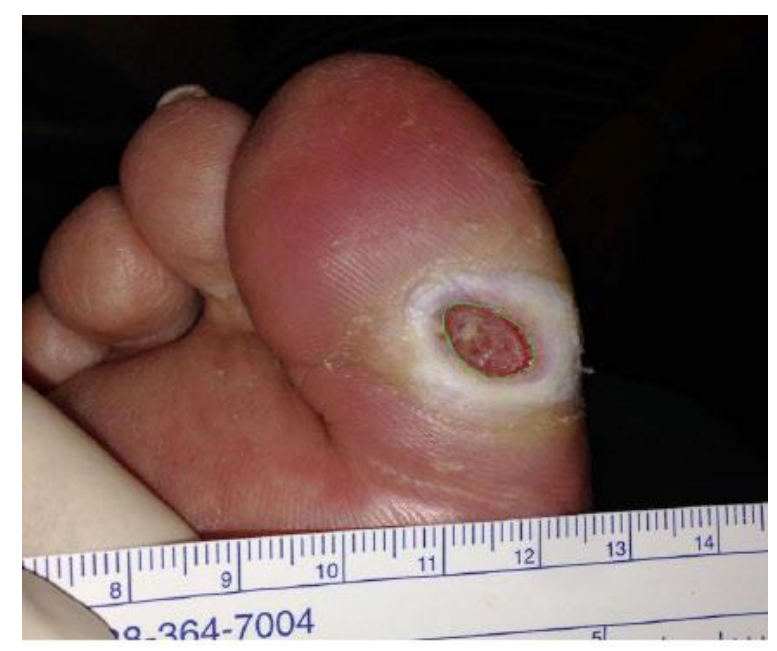

Fonte: Adaptado de Aragón-Sánchez et al., 2017).

Figura 3: Captura de tela da imagem incluindo a janela de medição.

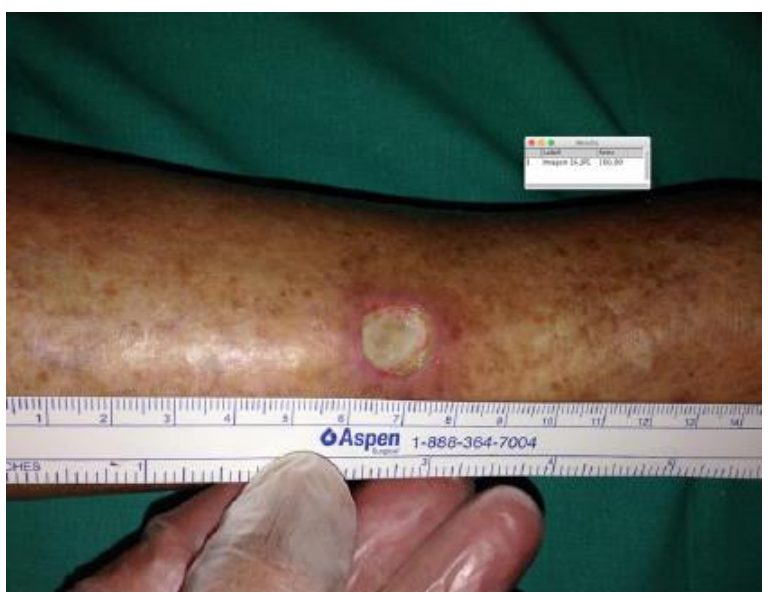

Fonte: Adaptado de Aragón-Sánchez et al., 2017). 


\section{Como citar este artigo}

Weber JF, Santos ALFdos. Utilização do software ImageJ para avaliar área de lesão dermonecrótica. [online], volume 4, n. 1. Editor responsável: Luiz Roberto de Oliveira. Fortaleza, mês e ano, p. 120-130. Disponível em: http://periodicos.ufc.br/resdite/index. Acesso em "dia/mês/ano".

Data de recebimento do artigo: 24/01/2019

Data de aprovação do artigo: 14/05/2019 